\title{
Potential for molecular-targeted therapy targeting human epidermal growth factor receptor-2 for invasive bladder cancer
}

\author{
YOSHIAKI YAMADA ${ }^{1}$, KATSUYA NARUSE $^{1}$, KOGENTA NAKAMURA $^{1}$, SHIGEYUKI AOKI $^{1}$, \\ TOMOHIRO TAKI $^{1}$, MOTOI TOBIUME ${ }^{1}$, KENJI ZENNAMI $^{1}$, REMI KATSUDA $^{1}$, \\ YASUSUKE INOUE $^{2}$, HIROSHI NOGUCHI ${ }^{2}$ and NOBUAKI HONDA ${ }^{1}$ \\ Departments of ${ }^{1}$ Urology and ${ }^{2}$ Emergency and Critical Care Medicine, \\ Aichi Medical University School of Medicine, Aichi, Japan
}

Received February 27, 2007; Accepted April 2, 2007

\begin{abstract}
Expression of human epidermal growth factor receptor-2 (HER-2/neu or HER-2) oncoprotein in invasive bladder cancer was examined by immunohistochemical staining in order to evaluate the potential for moleculartargeted therapy targeting HER-2 as a tailor-made treatment for patients with invasive bladder cancer. This study included 40 patients who were examined at Aichi Medical University Hospital and were pathologically diagnosed with invasive transitional cell carcinoma of the bladder (pT2 to pT4). Immunohistochemical staining using a Hercep test kit was performed to detect HER-2 expression, which was classified into four levels from 0 to $3+$ by two experienced pathologists, with $2+$ and $3+$ determined as positive. HER-2 staining in the primary tumor was determined as 0 in $9(22.5 \%)$ patients, $1+$ in $14(35 \%), 2+$ in $10(25 \%)$, and $3+$ in $7(17.5 \%)$, resulting in $17(17 / 40,42.5 \%)$ HER-2-positive patients. According to the classification of grade, one $(1 / 3,33.3 \%)$ grade 2 patient and $16(16 / 37,43.2 \%)$ grade 3 patients were HER-2 positive $(\mathrm{p}=0.99)$. According to the classification of stage, $12(12 / 22$, $54.5 \%)$ pT2 patients, $2(2 / 13,15.3 \%)$ pT3 patients, and 3 $(3 / 5,60 \%)$ pT4 patients were HER-2 positive $(\mathrm{p}=0.05)$. Lymph node metastasis was found in 10 patients, and $3(3 / 6$, $50 \%) \mathrm{pN} 2$ patients were HER-2 positive $(\mathrm{p}=0.32)$. There was a statistically significant difference between patients with HER-2-positive primary tumors and those with HER-2positive metastatic lymph nodes $(\mathrm{p}=0.02)$. This study suggested that $42.5 \%$ of patients with invasive bladder cancer
\end{abstract}

Correspondence to: Dr Yoshiaki Yamada, Department of Urology, Aichi Medical University School of Medicine, Nagakute, Aichi 480-1195, Japan

E-mail: 101959@gk.amu.aichi-med-u.ac.jp

Key words: invasive bladder cancer, human epidermal growth factor receptor-2, immunohistochemistry, molecular-targeted therapy may benefit from molecular-targeted therapy targeting HER-2, and that the efficacy of molecular-targeted therapy can be expected even for patients with lymph node metastases as long as their primary tumors are HER-2 positive.

\section{Introduction}

Currently, the standard treatment for invasive bladder cancer without metastasis is considered to be radical cystectomy. However, the results of radical cystectomy are substantially influenced by the pathological stage and the presence or absence of lymph node metastasis at the time of surgery. The cause-specific survival rate has been reported to be favorable at $\sim 70 \%$ when invasion was observed only in the tunica muscularis (pT2), whereas the rate was $30-40 \%$ when invasion into the surrounding adipose tissue was observed (pT3), and $\sim 20 \%$ when lymph node metastasis was noted (1-3). In addition, after radical cystectomy, most recurrences were distant metastases, with only $\sim 10 \%$ being local recurrences (4). Therefore, it is more important to eradicate such micrometastases that cannot be identified by imaging rather than to increase local curability to improve the treatment results of radical cystectomy. Surgical resection is not sufficient for invasive bladder cancer with involvement outside the bladder wall or lymph node metastasis, and additional therapy is required.

Multi-drug combination chemotherapy with anti-cancer agents is commonly used to treat progressive bladder cancer that is unresectable or metastatic. M-VAC therapy is a typical chemotherapy regimen and has been the gold standard since Sternberg et al $(5,6)$ reported a response rate of $72 \%$ and a complete remission rate of $36 \%$. However, subsequent studies have revealed that this therapy has a low response rate with a short duration of response, which lowers the prospect of long-term survival. In addition there is often concern about dose intensity because a large proportion of the targeted patients are elderly, and the high toxicity of the drug is a substantial physical burden to many elderly patients with bladder cancer $(7,8)$. Moreover, at present, the standard treatment for M-VAC-resistant cancer has not been established. Therefore, the development of novel regimens 
replacing M-VAC therapy is on-going, and clinical trials of anti-cancer agents, mainly gemcitabine and taxane, are being conducted. However, their effectiveness is still under examination (9-11).

On the other hand, molecular-targeted therapies for cancer are drugs developed to target changes in genes and molecules that are characteristic of cancer. Conventional anti-cancer agents primarily affect the process of nucleic acid, DNA, and microtubule synthesis and exert anti-tumor effects. These agents, however, have limited effectiveness due to poor tumor selectivity, since they affect both normal and tumor cells. For conventional anti-cancer agents, therefore, the maximum tolerated dose without toxicity has been regarded as the optimal dose. However, molecular-targeted therapeutic drugs generally have a weaker toxicity than anti-cancer agents. Moreover, these drugs can be administered safely even to elderly patients, which has been emphasized as an advantage. Furthermore, these drugs can be used in combination with existing anti-cancer agents, and are superior in that they can be used as a tailor-made treatment by coupling them with the development of biomarkers.

In this study, we retrospectively examined human epidermal growth factor receptor-2 (HER-2/neu or HER-2) oncoprotein expression by immunohistochemical (IHC) staining using a Hercep test kit (Dako Cystomention A/S, Carpinteria, CA, USA), in order to investigate the potential for molecular-targeted therapy targeting HER-2 as an effective treatment for patients with invasive bladder cancer.

\section{Materials and methods}

Patients. This study included 40 patients who were examined at Aichi Medical University Hospital from January 2001 to December 2004, and were pathologically diagnosed with invasive bladder transitional cell carcinoma (pT2 to pT4). The diagnostic methods were radical cystectomy for 33 patients, and TUR-Bt for 7 . The patients were $47-80$ years old (median, 65; mean, 65.6 \pm 8.4 years), and 37 were male and 3 were female. The pathological grades were grade 2 and 3 in 3 and 37 patients, respectively. The stages were pT2, pT3 and pT4 in 29, 5, and 6 patients, respectively. The $\mathrm{N}$ classifications were $\mathrm{pN} 0, \mathrm{pN} 1, \mathrm{pN} 2$ and $\mathrm{pNx}$ in $23,4,6$, and 7 patients, respectively. The histopathological grades were based on the World Health Organization (WHO) classification (12), and the TNM classification (13) was used to evaluate primary tumors and lymph node metastases.

This study was approved by the Institutional Review Board at Aichi Medical University (no. 275), and every patient gave consent after being sufficiently informed before the HER-2 oncoprotein staining. Table I shows the characteristics of the 40 patients.

Immunohistochemical staining and evaluation method. Paraffin sections, 5- $\mu \mathrm{m}$ thick, from bladder cancer samples taken during surgery were stained for HER-2 protein expression using a standardized Hercep test kit. The primary antibody in this assay was an affinity-purified rabbit polyclonal antiserum raised against an intracellular epitope of the human HER-2 molecule (Specification sheet: K5205, Dako, USA). The staining procedure included an antigen
Table I. Patient characteristics.

\begin{tabular}{lc}
\hline Number of patients & 40 \\
Age & $47-80$ \\
Average age & $65.6 \pm 8.35$ \\
Median age & 65 \\
Gender & \\
Male & 37 \\
Female & 3 \\
T classification & \\
pT2 & 22 \\
pT3 & 13 \\
pT4 & 5 \\
N classification & \\
pN0 & 23 \\
pN1 & 4 \\
pN2 & 6 \\
pNx & 7 \\
Grade & \\
G2 & 37 \\
G3 & 37 \\
\hline
\end{tabular}

retrieval step consisting of a 40-min incubation in $\mathrm{pH} 6.0$ citrate buffer (Target Retrieval, Dako) in a $99^{\circ} \mathrm{C}$ water bath. Staining was performed using an automated staining apparatus for IHC staining (Autostainer, Dako) according to the manufacturer's guidelines. The slides were counterstained with hematoxylin. Each IHC experiment included a set of control slides. For the negative control, the primary antibody was replaced with an irrelevant, isotype-matched antibody to control for nonspecific binding of the secondary antibody reagent. The positive control slide consisted of sections of cell blocks of three breast cancer cell lines, SKBR 3, MDAMB-175, and MDA-MB-231, which expressed 2.4 million, 92,000 and 22,000 HER-2 receptor molecules by Scatchard analysis, respectively, and corresponded to HER-2 scores of $3+, 1+$ and 0 , respectively. Histopathologic evaluation was performed, and the staining results were interpreted by two experienced pathologists and graded into four levels according to the classification of Carter et al (14). We considered scores of $>2+$ as positive (Fig. 1).

For statistical analysis, the Statistical Package for the Social Sciences (SPSS, Chicago, IL, USA) version 10.0 for Windows software was used. The Chi-square test was used to detect statistically significant differences between the groups, with a significance level of $\mathrm{p}<0.05$.

\section{Results}

HER-2 staining in the primary tumors was determined to be 0 in $9(22.5 \%)$ patients, $1+$ in $14(35 \%), 2+$ in $10(25 \%)$, and $3+$ in $7(17.5 \%)$. When $2+$ and $3+$ combined were determined as HER-2 overexpression, $17(17 / 40,42.5 \%)$ patients were HER-2 positive. According to the classification of grade, one $(1 / 3,33.3 \%)$ grade 2 patient and $16(16 / 37,43.2 \%)$ grade 3 patients were HER-2 positive. According to the classification 


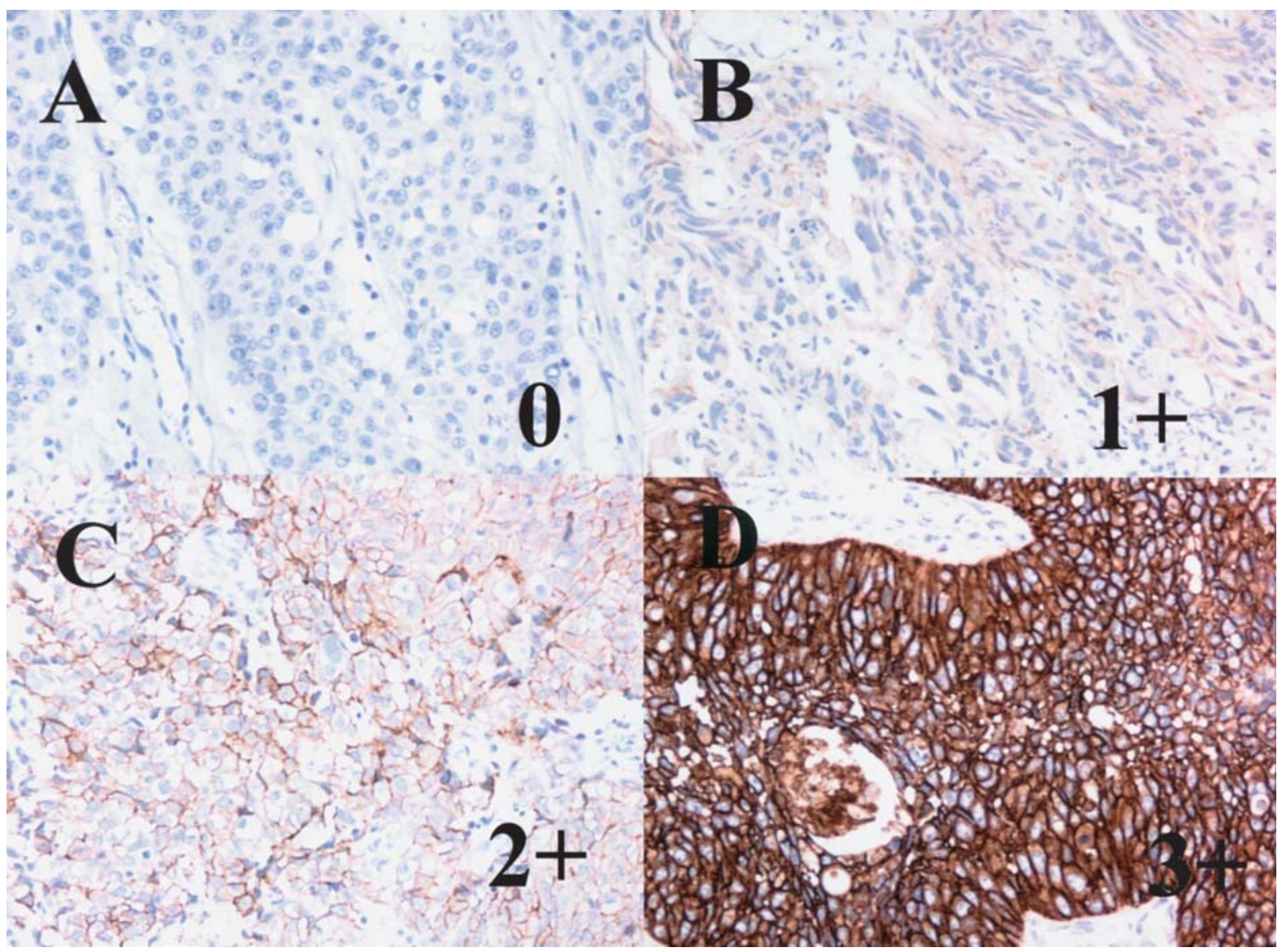

Figure 1. HER-2 expression in four primary tumors detected by immunohistochemistry using the Hercep test kit. (A) No staining or membrane staining observed in $<10 \%$ of tumor cells (HER-2 score 0); x400. (B) Faint or barely perceptible membrane staining detected in $>10 \%$ of tumor cells; cells are stained only in part of their membrane (HER-2 score 1+); $x 400$. (C) Weak to moderate, complete membrane staining observed in $>10 \%$ of tumor cells (HER-2 score $2+$ ); $x 400$. (D) Strong complete membrane staining observed in $>10 \%$ of tumor cells (HER-2 score $3+$ ); $x 400$.

Table II. Results by grade and stage.

\begin{tabular}{|c|c|c|c|c|}
\hline & HER-2 positive & HER-2 negative & Total & p-value \\
\hline \multicolumn{5}{|c|}{ Grade } \\
\hline $\mathrm{G} 2$ & $1(33.3 \%)$ & $2(66.6 \%)$ & 3 & \\
\hline G3 & $16(43.2 \%)$ & $21(56.8 \%)$ & 37 & $\mathrm{p}=0.99$ \\
\hline \multicolumn{5}{|c|}{$\mathrm{T}$ classification } \\
\hline $\mathrm{T} 2$ & $12(54.5 \%)$ & $10(45.4 \%)$ & 22 & \\
\hline T3 & $2(15.3 \%)$ & $11(84.6 \%)$ & 13 & \\
\hline $\mathrm{T} 4$ & $3(60.0 \%)$ & $2(40.0 \%)$ & 5 & $\mathrm{p}=0.05$ \\
\hline \multicolumn{5}{|c|}{$\mathrm{N}$ classification } \\
\hline N1 & $0(0 \%)$ & $4(100 \%)$ & 4 & \\
\hline $\mathrm{N} 2$ & $3(50 \%)$ & $3(50 \%)$ & 6 & $\mathrm{p}=0.32$ \\
\hline
\end{tabular}

of stage, $12(12 / 22,54.5 \%)$ pT2 patients, $2(2 / 13,15.3 \%)$ pT3 patients, and $3(3 / 5,60 \%)$ pT4 patients were HER-2 positive. Lymph node metastasis was observed in 10 patients, and $3(3 / 6,50 \%)$ pN2 patients were HER-2 positive. There was no statistically significant difference among the classifications. However, there was a significant trend in the stage classification although a statistically significant difference was not observed $(p=0.05)$ (Table II). In metastatic lymph nodes, HER-2 staining was determined as 0 in $7(70 \%)$ patients and $3+$ in $3(30 \%)$, with no patient having $1+$ or $2+$. In addition, all primary tumors were grade 3 , and HER-2 staining was determined as $3+$ in all patients with HER-2-positive lymph nodes. There was a statistically significant difference between patients with HER-2-positive primary tumors and those with HER-2-positive metastatic lymph nodes $(\mathrm{p}=0.02)$ (Table III). 
Table III. HER-2 expression in primary tumors and lymph node metastases.

\begin{tabular}{lcc}
\hline & \multicolumn{2}{c}{ Primary tumors } \\
\cline { 2 - 3 } & Positive & Negative \\
\hline Lymph node metastases & 3 & 0 \\
Positive & 0 & 7 \\
Negative & & \\
\hline
\end{tabular}

$\mathrm{p}=0.02$. Patients with lymph node metastases $(\mathrm{n}=10)$.

\section{Discussion}

HER-2/neu (or HER-2) is a proto-oncogene located at chromosome $17 \mathrm{q} 21$, encoding a protein with a molecular weight of $185 \mathrm{kDa}$ which has a transmembrane receptor structure. This protein is a tyrosine kinase-type cell surface receptor which has amino acid sequences similar to those of an epidermal growth factor receptor, and that cell differentiation and proliferation are stimulated by a ligand binding to an extracellular domain. Moreover, fundamental studies have shown that HER-2 overexpression induces cell transformation and that HER-2-positive tumors are more aggressive. With regard to the distribution of HER-2 in normal tissues, HER-2 is slightly expressed only in the liver, bile duct, gastrointestinal tract, skin, genital organs and urinary tract, with limited expression in most normal tissues (15-17). Therefore, the potential for molecular-targeted therapy targeting HER-2 is of great interest.

Since Slamon et al (18) reported in 1987 the correlation between HER-2 (c-erbB-2) gene amplification and breast cancer with a poor outcome, HER-2 has been considered to be a poor prognostic factor in breast cancer. In addition, breast cancer with HER-2 overexpression has been reported to be resistant to hormone therapy $(19,20)$, resulting in the establishment of treatment with trastuzumab (21). Currently, the methods to analyze HER-2 in tissues include analysis of gene amplification, mRNA overexpression, and protein overexpression; however, possible methods for use on formalinfixed paraffin sections are IHC and fluorescent in situ hybridization (FISH).

Examination with gene amplification rather than with antigen expression is a more reliable method to identify patients with HER-2-positive breast cancer (22). However, it is inconclusive whether the present results can similarly be applied to patients with cancer other than breast cancer, for example, patients with bladder cancer. Moreover, based on the report by Jacobs et al (23) which showed that the positive agreement rate was $91.1 \%$ by both IHC and FISH in 100 patients with breast cancer, we used the cost-effective IHC for analysis in this study.

In our study, the Dako Hercep test kit was used. In studies using rabbit polyclonal antibodies as those employed in the Dako Hercep test kit as the primary antibody, Sauter et al (24) reported HER-2 overexpression in 61 (43\%) out of 141 patients. Underwood et al (25) reported HER-2 expression in
$45(51 \%)$ out of 89 patients with recurrence and in $9(45 \%)$ out of 20 patients without recurrence. Furthermore, according to the report by Gardmark et al (26), 55\% (47/86) of primary tumors were HER-2 positive. These findings appear to be consistent with the present result that $42.5 \%$ (17/40) of patients were HER-2 positive. However, Wester et al (27) conducted a study on 21 patients with bladder cancer with metastasis and reported that $81 \%(17 / 21)$ of the primary tumors were HER-2 positive. This high frequency of HER-2 expression was attributed to the fact that the study population consisted of patients with known metastatic status and all tumors had a high grade of atypism. In addition, Miyamoto et al (28) and Sato et al (29) reported an important correlation between an increase in HER-2 expression and grade of atypism. However, there was no significant difference among grades in our study, likely due to the limited number of patients (3) with grade 2 .

There are few reports on HER-2 and lymph node metastasis in bladder cancer. Gardmark et al (26) reported that $40 \%$ $(34 / 86)$ of lymph node metastases were HER-2 positive and $72 \%(34 / 47)$ also had HER-2-positive primary tumors. In addition, Wester et al (27) reported that 67\% (14/21) of metastatic lesions were HER-2 positive, and all patients with HER-2-positive metastatic lesions also had HER-2-positive primary tumors. However, they also reported that 3 patients (14\%) had HER-2-negative metastatic lesions despite having HER-2-positive primary tumors. Moreover, they reported that $8(89 \%)$ out of 9 patients with local lymph node metastases were HER-2 positive as compared to $6(50 \%)$ HER-2-positive patients among 12 with distant metastases, and they stated that HER-2 expression tended to vary between local and distant metastases. This finding is notable, and we think it should be examined in a larger number of patients. Our results also showed that 30\% (3/10) had HER-2positive metastatic lymph nodes; these 3 patients also had HER-2-positive primary tumors. In this study, we did not search for HER-2 expression in patients having no metastasized lymph nodes, although HER-2 expression can also be seen in patients with negative lymph node metastases. Therefore, patients with negative lymph nodes should also be studied in the future.

Different results may be produced as a result of differences in procedures to evaluate HER-2 overexpression, including the storage conditions of antigens, types of antibodies used, and methods for antigen retrieval when investigating HER-2 expression in bladder cancer. Thus, we believe that a consistent method should be used for evaluation. We performed IHC using a Dako Autostainer to avoid procedural differences as much as possible in this study. However, further study is required to determine whether our results can be applied to bladder cancer, because the positive controls for the Hercep test kit are breast cancer cell lines. We, however, used this kit employing the determination method described by Carter et al (14), since detection of HER-2 using the Hercep test kit is a criterion to predict the therapeutic effectiveness of trastuzumab for breast cancer.

Trastuzumab is a human monoclonal antibody against the outer cell membrane of the HER-2 receptor. By binding to the receptor, this drug blocks subsequent signals to the receptor, inhibits cancer growth, and produces an anti-tumor effect, 
representing a molecular-targeted therapeutic drug targeting HER-2. Our results suggest that $42.5 \%$ of patients with invasive bladder cancer may benefit from this drug, and that the efficacy of this drug can be expected even in patients with lymph node metastases, as long as their primary tumors are HER-2 positive. Further clinical studies on various phases are needed to establish an effective treatment with trastuzumab for bladder cancer.

\section{References}

1. Bassi P, Ferrante GD, Piazza N, et al: Prognostic factors of outcome after radical cystectomy for bladder cancer: a retrospective study of a homogeneous patient cohort. J Urol 161: 1494-1497, 1999.

2. Ghoneim MA, el-Mekresh MM, el-Baz MA, el-Attar IA and Ashamallah A: Radical cystectomy for carcinoma of the bladder: critical evaluation of the results in 1,026 cases. J Urol 158: 393-399, 1997.

3. Waehre H, Ous S, Klevmark B, et al: A bladder cancer multiinstitutional experience with total cystectomy for muscleinvasive bladder cancer. Cancer 72: 3044-3051, 1993.

4. Schuster TG, Smith DC and Montie JE: Pelvic recurrences post cystectomy: current treatment strategies. Semin Urol Oncol 19: 45-50, 2001.

5. Sternberg CN, Yagoda A, Scher HI, et al: Preliminary results of M-VAC (methotrexate, vinblastine, doxorubicin and cisplatin) for transitional cell carcinoma of the urothelium. J Urol 133: 403-407, 1985.

6. Sternberg CN, Yagoda A, Scher HI, et al: Methotrexate, vinblastine, doxorubicin, and cisplatin for advanced transitional cell carcinoma of the urothelium. Efficacy and patterns of response and relapse. Cancer 64: 2448-2458, 1989.

7. Loehrer PJ Sr, Einhorn LH, Elson PJ, et al: A randomized comparison of cisplatin alone or in combination with methotrexate, vinblastine, and doxorubicin in patients with metastatic urothelial carcinoma: a cooperative group study. J Clin Oncol 10: 1066-1073, 1992.

8. Saxman SB, Propert KJ, Einhorn LH, et al: Long-term followup of a phase II intergroup study of cisplatin alone or in combination with methotrexate, vinblastine, and doxorubicin in patients with metastatic urothelial carcinoma: a cooperative group study. J Clin Oncol 15: 2564-2569, 1997.

9. Bajorin DF, McCaffrey JA, Dodd PM, et al: Ifosfamide, paclitaxel, and cisplatin for patients with advanced transitional cell carcinoma of the urothelial tract: final report of a phase II trial evaluating two dosing schedules. Cancer 88: 1671-1678, 2000.

10. von der Maase H, Hansen SW, Roberts JT, et al: Gemcitabine and cisplatin versus methotrexate, vinblastine, doxorubicin, and cisplatin in advanced or metastatic bladder cancer: results of a large, randomized, multinational, multicenter, phase III study. J Clin Oncol 18: 3068-3077, 2000.

11. von der Maase H, Sengelov L, Roberts JT, et al: Long-term survival results of a randomized trial comparing gemcitabine plus cisplatin, with methotrexate, vinblastine, doxorubicin, plus cisplatin in patients with bladder cancer. J Clin Oncol 23: 4602-4608, 2005.
12. Epstein JI, Amin MB, Reuter VR and Mostofi FK: The World Health Organization/International Society of Urological Pathology Consensus Classification of Urothelial (transitional cell) Neoplasms of the Urinary Bladder. Am J Surg Pathol 22: 1435-1448, 1998.

13. Sobin LH and Wittekind $\mathrm{CH}$ (eds): TNM classification of malignant tumors. 6th edition. Wiley-Liss, New York, pp199-202, 2002.

14. Carter P, Presta L, Gorman CM, et al: Humanization of an antip185HER2 antibody for human cancer therapy. Proc Natl Acad Sci USA 89: 4285-4289, 1992.

15. Natali PG, Nicotra MR, Bigotti A, et al: Expression of the p185 encoded by HER 2 oncogene in normal and transformed human tissues. Int J Cancer 45: 457-461, 1990.

16. Press MF, Cordon-Cardo C and Slamon DJ: Expression of the HER-2/neu proto-oncogene in normal human adult and fetal tissues. Oncogene 5: 953-962, 1990.

17. Gorgoulis VG, Barbatis C, Poulias I and Karameris AM: Molecular and immunohistochemical evaluation of epidermal growth factor receptor and c-erb-B-2 gene product in transitional cell carcinomas of the urinary bladder: a study in Greek patients. Mod Pathol 8: 758-764, 1995.

18. Slamon DJ, Clark GM, Wong SG, Levin WJ, Ullrich A and McGuire WL: Human breast cancer: correlation of relapse and survival with amplification of the HER-2/neu oncogene. Science 235: 177-182, 1987.

19. Slamon DJ, Godolphin W, Jones LA, et al: Studies of the HER$2 /$ neu proto-oncogene in human breast and ovarian cancer. Science 244: 707-712, 1989.

20. Press MF, Bernstein L, Thomas PA, et al: HER-2/neu gene amplification characterized by fluorescence in situ hybridization: poor prognosis in node-negative breast carcinomas. J Clin Oncol 15: 2894-2904, 1997.

21. Slamon DJ, Leyland-Jones B, Shak S, et al: Use of chemotherapy plus a monoclonal antibody against HER 2 for metastatic breast cancer that overexpresses HER2. N Engl J Med 344: 783-792, 2001.

22. Mass R, Sanders C, Charlene K, Johnson L, Everett T and Anderson S: The concordance between the clinical trial assay (CTA) and fluorescence in situ hybridization (FISH) in the herceptin pivotal trials. Proc Am Soc Clin Oncol 19: 291, 2000.

23. Jacobs TW, Gown AM, Yaziji H, Barnes MJ and Schnitt SJ: Comparison of fluorescence in situ hybridization and immunohistochemistry for the evaluation of HER-2/neu in breast cancer. J Clin Oncol 17: 1974-1982, 1999.

24. Sauter G, Moch H, Moore D, et al: Heterogeneity of erbB-2 gene amplification in bladder cancer. Cancer Res 53: 2199-2203, 1993.

25. Underwood M, Bartlett J, Reeves J, Gardiner DS, Scott R and Cooke T: C-erbB-2 gene amplification: a molecular marker in recurrent bladder tumors? Cancer Res 55: 2422-2430, 1995

26. Gardmark T, Wester K, de la Torre M, Carlsson J and Malmstrom PU: Analysis of HER2 expression in primary urinary bladder carcinoma and corresponding metastases. BJU Int 95: 982-986, 2005.

27. Wester K, Sjostrom A, de la Torre M, Carlsson J and Malmstrom PU: HER-2 - a possible target for therapy of metastatic urinary bladder carcinoma. Acta Oncol 41: 282-288, 2002.

28. Miyamoto H, Kubota Y, Noguchi S, et al: C-ERBB-2 gene amplification as a prognostic marker in human bladder cancer. Urology 55: 679-683, 2000.

29. Sato K, Moriyama M, Mori S, et al: An immunohistologic evaluation of C-erbB-2 gene product in patients with urinary bladder carcinoma. Cancer 70: 2493-2498, 1992. 